\title{
Nonperturbative fragmentation of the top quark
}

\author{
A. Dobado \\ Departamento de Física Teórica and Instituto de Física Fundamental, Universidad Complutense de Madrid, \\ 28040 Madrid, Spain \\ M. Urdiales \\ Departamento de Física Teórica, Universidad Autonóma de Madrid, 28049 Madrid, Spain
}

(Received 2 May 1991)

\begin{abstract}
By the use of a simple phenomenological string model, the energy lost by the top quark through nonperturbative fragmentation effects before its weak decay is computed. This energy lost is in first approximation proportional to the rate between the decay time and the hadronization time of the top as measured in the center-of-mass frame of the top and its color partner. Precise computations of the energy lost by the top in terms of its mass when produced in colliders such as the Fermilab Tevatron, CERN Large Hadron Collider, Superconducting Super Collider, and CERN Linear Collider are presented. As expected, this effect decreases quickly with the top mass but it could be relevant for a top mass below 100 $\mathrm{GeV}$.
\end{abstract}

\section{INTRODUCTION}

According to our usual understanding of QCD, quarks and gluons cannot appear as observable final states of reactions because of confinement. For this reason, when they are produced in high-energy reactions, they suffer an extremely complex process called fragmentation or hadronization. As a result of this fragmentation, quarks and gluons are transformed in a large number of hadrons resulting in the entropy of the whole system being greatly increased.

Nevertheless, this picture can be completely different for heavy enough quarks, especially the top. As the quark mass is getting heavier, the weak decay width becomes larger and larger because the available phase space increases quickly with the quark mass. For instance, for a heavy enough top quark, its weak decay width to a real $W$ and a $b$ quark is given roughly by $\Gamma_{0} \sim 2.6$ $\operatorname{GeV}\left(m_{t} / 200\right)^{3}$. Therefore, if the top quark is very heavy, its mean life is so short that the fragmentation process does not have time to take place before the top decay and it is relegated to the bottom quark.

Between the two extreme situations (complete fragmentation or no fragmentation at all) we have a lot of intermediate possibilities. Nevertheless, recent experimental lower bounds on the top mass $\left(m_{t} \sim 90 \mathrm{GeV}[1]\right)$ suggest the real case may be very close to the nofragmentation case. In fact, at least to the knowledge of the authors, all the phenomenological predictions concerning top production and detection in present and future accelerators are based on a pure weak decay description of the top signature. Of course, the situation remains unclear and it is very likely that some amount of fragmentation could accompany the top decay, especially for top masses close to the present bound. Having a more precise idea of what will be the effect of the top fragmentation on its signature is important for two reasons. First, if the top quark produces several hadrons before its decay, the experimental signal of the events could be more obscure and the discovery of the top at the Fermilab Tevatron will become even harder if fragmentation effects are not properly taken into account. Second, at the CERN Large Hadron Collider (LHC) and Superconducting Super Collider (SSC) [or maybe at CERN Linear Collider (CLIC)] where larger statistics of top events is expected, fragmentation effects could produce systematical errors in the measure of top parameters such as the mass and the width, since part of its energy and momentum could be transferred to the hadrons produced in the fragmentation process.

To bring some light on this problem, the hadronization and decay times for $t \bar{q}$ systems were compared by Bigi et $a l$, in [2] and they concluded that, for a top mass $\left(m_{t}\right)$ larger than about $100 \mathrm{GeV}$, nonperturbative fragmentation effects can be neglected because these $t \bar{q}$ states do not have time to be formed before the decay of the top. They stated that the relative amount of energy residing in the remnant jet goes to zero when the top mass increases and they also pointed out the importance of perturbative top fragmentation, which will not be considered in our work.

More recently [3] Orr and Rosner have put the emphasis on the fact that both the hadronization and the decay time are affected by the motion of the quarks and, hence, that nonperturbative effects can or cannot be ignored above a particular mass is an energy-dependent statement (in fact this was also taken into account in [2]). Accordingly, for any value of the top mass we can always find a center-of-mass energy for the process producing the top so that nonperturbative fragmentation effects are present. Orr and Rosner illustrated this point by considering in detail the case of $e^{+} e^{-}$going to $t \bar{t}$ and finding large values of the rate, decay time, and hadronization time, for reasonable values of the top mass and the center-of-mass energy of the reaction. In this work we extend their analysis in two different lines. First, we consider also $\bar{p} p$ and $p p$ reactions. This is an important ex- 
tension since it is in this kind of processes where we expect to discover and produce massively the top quark (Tevatron, LHC, and SSC). On the other hand, we will also try to compute the energy transferred from the top quark to the hadrons in the fragmentation process. We think this energy gives a precise notion of how relevant the nonperturbative fragmentation of the top quark could be and how it could disturb the expected signature of the events.

To compute this energy lost by nonperturbative fragmentation effects we will use a simple model in the same spirit of the Lund model of hadronization [4]. This model has proved to give a simple description of many of the complex nonperturbative phenomena involved in hadronization and it made successful predictions of effects such as gluon-(anti)quark color correlations in $e^{+} e^{-}$reactions which were confirmed experimentally. In the present work, we will describe the nonperturbative gluon fields (flux tubes) by the use of a string connecting the top quark and its color partner. As in the Lund model we will reduce the problem to one dimension (the longitudinal one) which is relevant in high-energy processes and we will find a simple equation giving the energy lost by the top quark and transferred to the string, i.e., at the hadrons produced in the fragmentation process.

The plan of the paper will be the following. In Sec. II we introduce in detail and solve the above-mentioned string model of top fragmentation. In Sec. III we obtain the cross section for the production of the different color flux structures that can appear in $e^{+} e^{-}, p p$, and $p \bar{p}$ reactions giving $t \bar{t}$ pairs. In Sec. IV we give some details about the way the computations were done and the numerical results obtained which are relevant for Tevatron, LHC, SSC, and CLIC. Finally, in Sec. V we comment on these results and give some experimental prospects.

\section{THE STRING PICTURE OF TOP FRAGMENTATION}

In order to make an estimation of the energy lost by the top quark due to nonperturbative fragmentation effects from its creation to its decay we will consider the following simple model. We will assume that the color flux tube can be described by a massless bosonic string in the same philosophy of the Lund model [4]. The possibility of describing the gluon flux tube at large distances as a bosonic string is not clear at all since other kinds of string are possible in principle. Nevertheless, for the sake of simplicity we will adopt this picture in this work with the hope that our numerical results will not be very dependent on this special choice.

At one of the ends of the string (say 1 ) we will attach a top quark of mass $m_{1}=m_{t}$. In fact, $m_{1}$ should be taken to be equal to $\sqrt{m_{t}^{2}+p_{T}^{2}}$ with $p_{T}$ being the typical transverse momentum in hadronization processes. As this $p_{T}$ is much smaller than the top mass $m_{t}$ we will neglect this correction. The object to be attached to the other end of the string (say 2) will be process dependent. For instance, in the case of top production in $e^{+} e^{-}$reactions it will be the corresponding antitop. In $p p$ reactions, the object at the other side of the string will be a color octet. In fact, this octet represents the rest of the proton once a gluon has been extracted from it. In the $p \bar{p}$ case, as there is also a contribution from $q \bar{q}$ initial states, a color triplet has to be taken into account, too.

Therefore, in $p p$ and $p \bar{p}$ reactions the produced top quark is not connected through the string with the antitop but with the destroyed proton or antiproton that typically produces a very energetic jet in the beam direction. For the sake of simplicity we will consider this rest of the (anti)proton as a unique particle of mass $m_{2}$ located at the second end of the string. With these assumptions the system to be considered is just a bosonic string with masses $m_{1}$ and $m_{2}$ attached at its ends. This kind of system was first studied in [5] in the $m_{1}=m_{2}$ case. For the sake of completeness we will present here the simple extension to the general case, which is the one interesting for us.

A point of the world sheet of the color flux string will be given by $x \equiv X^{\mu}(\tau, \sigma), \sigma$ and $\tau$ being arbitrary parameters spacelike and timelike, respectively, with $\tau \in\left[\tau_{1}, \tau_{2}\right]$ and $\sigma \in[0, \pi]$. The action describing the dynamics of the string with massive ends will be taken to be

$S=-\int_{\tau_{1}}^{\tau_{2}} d \tau\left[\int_{0}^{\pi} d \sigma \mathcal{L}\left(\dot{x}, x^{\prime}\right)+L_{1}\left(\dot{x}_{1}\right)+L_{2}\left(\dot{x}_{2}\right)\right]$,

where $\quad x^{\prime} \equiv \partial x / \partial \sigma, \dot{x} \equiv \partial x / \partial \tau, x_{1} \equiv X_{\mu}(\tau, 0), \quad$ and $\quad x_{2}$ $\equiv X_{\mu}(\tau, \pi)$ are the ends of the string.

The Lagrangians are given by

$$
\begin{aligned}
\mathcal{L}\left(\dot{x}, x^{\prime}\right) & =-T\left[\left(\dot{x} x^{\prime}\right)^{2}-\dot{x}^{2} x^{\prime 2}\right]^{1 / 2}, \\
L_{1} & =-m_{1}\left(-\dot{x}_{1}^{2}\right)^{1 / 2}, \\
L_{2} & =-m_{2}\left(-\dot{x}_{2}^{2}\right)^{1 / 2} .
\end{aligned}
$$

Here $T$ is the string tension, $m_{1}$ and $m_{2}$ are the masses of the ends, and the signature $(-,+,+,+)$ is taken. By the use of the standard method the following motion equations are obtained:

$$
\begin{aligned}
& \frac{\partial \mathcal{L}}{\partial x^{\prime}}+\frac{d}{d \tau} \frac{d L_{1}}{d \dot{x}_{1}}=0, \quad \sigma=0, \\
& \frac{\partial \mathcal{L}}{\partial x^{\prime}}-\frac{d}{d \tau} \frac{d L_{2}}{d \dot{x}_{2}}=0, \quad \sigma=\pi, \\
& \frac{d}{d \sigma} \frac{\partial \mathcal{L}}{\partial x^{\prime}}+\frac{d}{d \tau} \frac{\partial \mathcal{L}}{\partial \dot{x}}=0, \quad \sigma \in(0, \pi) .
\end{aligned}
$$

By the use of the reparametrization invariance of the action it is possible to choose the timelike gauge where $x^{0}(\tau, \sigma)=\tau=t$. In addition, we will go further in the set of simplifications we are adopting and we will consider only the longitudinal degree of freedom of the string. Of course, this is the most relevant one in the fragmentation of high-energy colored objects. Although the transversal degrees of freedom can play an important role in the description of some properties of the jets, they can be neglected in a first approximation since we are interested only in the computation of the energy lost by fragmentation effects. Accordingly we will use only one spatial coordinate $z=X^{1}(t, \sigma)$. In this case the equations of motion read 


$$
\begin{aligned}
& T \frac{z^{\prime}}{\left|z^{\prime}\right|}-m_{1} \frac{d}{d t} \frac{\dot{z}_{i}}{\sqrt{1-\dot{z}_{1}^{2}}}=0, \quad \sigma=0, \\
& T \frac{z^{\prime}}{\left|z^{\prime}\right|}+m_{2} \frac{d}{d t} \frac{\dot{z}_{2}}{\sqrt{1-\dot{z}_{2}^{2}}}=0, \quad \sigma=\pi, \\
& \frac{d}{d \sigma} \frac{z^{\prime}}{\left|z^{\prime}\right|}=0, \quad \sigma \in(0, \pi),
\end{aligned}
$$

where $z_{1}(t)=z(t, 0)$ and $z_{2}(t)=z(t, \pi)$.

The last equation means that the sign of $z^{\prime}$ does not depend on $\sigma$. By using again reparametrization invariance we can choose $z^{\prime}(t, \sigma)$ independent of $\sigma$, so that

$$
\int_{0}^{\pi} d \sigma\left|z^{\prime}\right|=\left|z_{2}-z_{1}\right|
$$

where $z^{\prime} /\left|z^{\prime}\right|=\operatorname{sgn}\left(z_{2}-z_{1}\right)$. Assuming, for instance $z_{2}>z_{1}$, we obtain

$$
\begin{aligned}
& \frac{m_{1}}{T} \frac{d}{d t} \frac{\dot{z}_{1}}{\sqrt{1-\dot{z}_{1}^{2}}}=1, \\
& -\frac{m_{2}}{T} \frac{d}{d t} \frac{\dot{z}_{2}}{\sqrt{1-\dot{z}_{2}^{2}}}=1 .
\end{aligned}
$$

This equation could also be derived from the simple Hamiltonian

$$
H=\left(p_{1}^{2}+m_{1}^{2}\right)^{1 / 2}+\left(p_{2}^{2}+m_{2}^{2}\right)^{1 / 2}+T\left|z_{2}-z_{1}\right|,
$$

$p_{1}$ and $p_{2}$ being the momenta of the ends of the string.

To integrate the above equations we select the initial conditions appropriate for the physical situation that we want to describe, i.e., $z_{1}(0)=z_{2}(0)=0$ and $p_{0}=-p_{1}(0)$ $=p_{2}(0)>0$. This choice corresponds to the creation of a zero long string at $t=0$ with the ends going back to back with equal absolute values of its momenta. This last condition means that we are describing the problem in the string's center-of-mass frame. Conventionally, we take the end 1 (the one of the top quark) as going to the left in a space-time diagram. With this initial condition it is straightforward to find the solution to the equations of motion:

$z_{1}(t)=\frac{m_{1}}{T}\left\{\left[1+\left(\frac{T t}{m_{1}}-\frac{p_{0}}{m_{1}}\right)^{2}\right]^{1 / 2}-\left(1+\frac{p_{0}^{2}}{m_{1}^{2}}\right)^{1 / 2}\right\}$,

$z_{2}(t)=-z_{1}(t) \quad\left(m_{1} \rightleftharpoons m_{2}\right)$,

and

$$
z(t, \sigma)=z_{1}(t)\left(1-\frac{\sigma}{\pi}\right)+z_{2}(t) \frac{\sigma}{\pi} .
$$

Note that for small $t$ we have assumed $z_{1}<z_{2}$. For large enough time this cannot be always the case because the motion of the string is oscillating (a yo-yo-mode). The time needed by the string to reach the maximum distance (the return time) between its ends is $t_{R}=p_{0} / T$. This time is much larger than the ones we are interested in, so we can safely use Eqs. (8) and (9) to describe the motion of the string and its ends. The dynamical interpretation of this motion is quite simple. At $t=0$ the top quark and its colored partner are created at the origin with very large opposite momenta. They start to separate but, doing this, they transfer some of their enormous kinetic energy to the string. The energy of the string (the term $T\left|z_{2}-z_{1}\right|$ in Eq. (7) will increase with time from its initial zero value. The physical meaning of the energy transferred by the end 1 (i.e., the top quark) to the string (i.e., the color-flux tube) is the energy lost by the top quark due to nonperturbative fragmentation effects. We expect this energy to be transformed in the hadrons that under some conditions must accompany the production of the top quark. Of course, the process will continue until the final decay of the top quark by weak interactions at a time $t_{d}$. Note that this decay time is much smaller than the return time, in the physical situation of interest (i.e., $\left.t_{d} \ll t_{R}\right)$. The energy lost by the top quark before its decay is the one we are interested in. Note also the different scales involved in the problem, i.e., the initial momentum of the top $p_{0}(\sim 1 \mathrm{TeV}$ in the interesting applications), the mass of the top, $m_{t} \sim 100 \mathrm{GeV}$, and the squared root of the string tension, $\sqrt{T} \sim 0.4 \mathrm{GeV}$, that sets the scale of nonperturbative effects in QCD. In fact, we have yet another independent scale which is the width of the top $\Gamma_{0} \sim 1 \mathrm{GeV}$.

As explained above, Eqs. (8) and (9) describe the expansion of the string for times $t$ smaller than the returning time $t_{R}$ but only provided that the string does not break. However, it is expected that when the energy of string is larger than some critical value, the string starts breaking. In fact, if fragmentation effects of the top quark have some relevance, the string has to break several times before the top decays at time $t_{d}$. In this situation, Eqs. (8) and (9) do not apply in principle because they do not take into account the different breakings that occur at times $t_{1}, t_{2}, \ldots, t_{n}<t_{d}$ (see Fig. 1). However, as these breakings are causally disconnected, the ends of the string are not affected by them until the time $t_{c}$ which typically is close to the return time $t_{R}$. As we are interested in situations with $t_{d}<<t_{R} \sim t_{c}$, we arrive at the conclusion that, in spite of the several breakings of the string, Eq. (8) describes the motion of the ends of the string until the decay of the top. This, of course, is not the case for Eq. (9) for obvious reasons.

Now, we are in condition to make the computation of the total energy transferred by the top quark to the string at time $t_{d}$ :

$$
\Delta E=E_{t}(0)-E_{t}\left(t_{d}\right),
$$

where $E_{t}(t)=E_{1}(t)$ is the top-quark energy as a function of time. From the discussion above, this energy (for $\left.t<t_{R}\right)$ is given by

$$
E_{t}(t)=\left[p_{1}^{2}(t)+m_{1}^{2}\right]^{1 / 2}=m_{1}\left[1-\dot{z}_{1}^{2}(t)\right]^{-1 / 2} .
$$

For the cases of practical interest we always have $T t \ll m_{1}$ so we can write Eq. (8) as

$$
z_{1}(t)=-\beta_{1}(0) t+\frac{T}{p_{0}}\left[\frac{m_{1}}{p_{0}}\right]^{2} \beta_{1}^{3}(0) \frac{t^{2}}{2},
$$

where $\beta_{1}(t)=\left|\dot{z}_{1}(t)\right|$. 


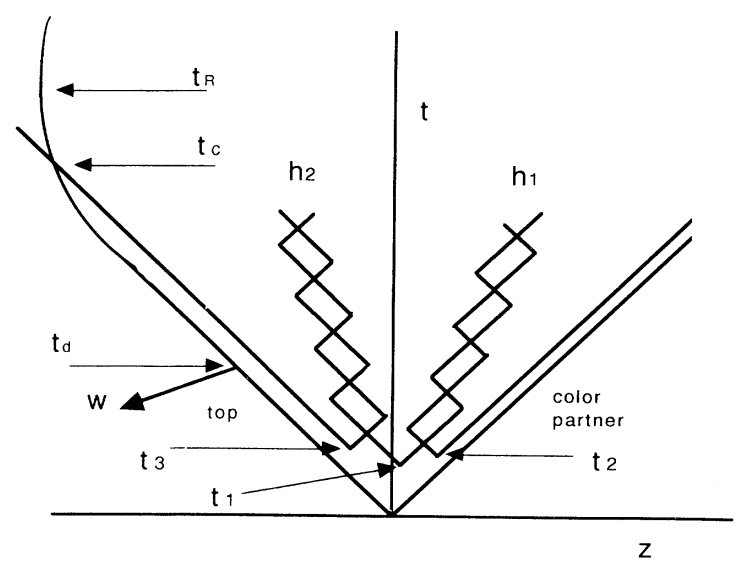

FIG. 1. Space-time diagram showing the different events involved in the creation, hadronization and decay of the top. $t_{1}$, $t_{2}$, and $t_{3}$ are different breaking times of the string, $t_{d}$ is the decay time of the top, $t_{c}$ is the time needed by the top, if it were stable, to know the strong is breaking, $t_{R}$ is the top returning time, and $h_{1}$ and $h_{2}$ are the first hadrons produced.

Therefore, in this approximation the top velocity decreases linearly with time. Finally, we obtain the simple result

$$
E_{1}(t)=E_{1}(0)-\beta_{1}(0) T t
$$

and Eq. (10) becomes

$$
\Delta E=\beta_{1}(0) T t_{d}
$$

Now we can define the hadronization time $t_{h}$ as the time required for the string to be $T^{-1 / 2}$ long, i.e.,

$$
t_{h}=\frac{T^{-1 / 2}}{\beta_{1}(0)+\beta_{2}(0)}
$$

in the first approximation. Note that this definition is the same as the one of [3] provided one defines their constant $\Lambda$ as $\Lambda^{2} \equiv T$. Therefore, Eq. (13) can be written as

$$
\Delta E=\frac{\beta_{1}(0)}{\beta_{1}(0)+\beta_{2}(0)} \sqrt{T} \frac{t_{d}}{t_{h}}=K \sqrt{T} \frac{t_{d}}{t_{h}} .
$$

In the case of top production in $p p$ or $p \bar{p}$ collisions $m_{2} \ll p_{0}$ typically so we can take $K=\beta_{1}(0) /\left[1+\beta_{1}(0)\right]$, but in $e^{+} e^{-}$collisions $\beta_{1}(0)=\beta_{2}(0)$ so $K=\frac{1}{2}$. Equation (16) is very simple but interesting because it relates the energy lost by the top quark due to nonperturbative effects and the rate between decay and hadronization time. Note that this rate was considered in [3] in the $e^{+} e^{-}$case as a measure of the fragmentation effects in top production and decay. Now we see that, under some well defined conditions, this rate is also a measure of the energy transferred by the top to the hadrons produced during its fragmentation.

At this point, some comments are in order. Until now, the description of the dynamics of the string and its ends has been completely classical and one could ask if it is possible to ignore quantum effects. The answer to this question was given some time ago by Low and Gottfried [6]. In this work they showed that the dynamics of the longitudinal degrees of freedom in hard reactions is essentially classical and, hence, a classical picture of the longitudinal evolution of the collision is appropriate. In particular, for a large energy particle $(E)$ we have the uncertainty relation $\Delta z \Delta y>1 / E, z$ being the longitudinal coordinate and $y$ the rapidity $\left(y=\frac{1}{2} \ln [(E+p) /(E-p)]\right.$ with $p$ being the particle momentum ).

Let us call now $x$ the fraction of the initial energy plus momentum of the top carried by the top hadron $t \bar{q}$ after the fragmentation process (this variable is usually called $z$ but we do not use this notation here to avoid confusion with the longitudinal coordinate). In principle, $x$ is a random variable described by some density of probability function $f(x)$, but in our classic and hence deterministic picture it has a well defined value $x_{\mathrm{cl}}$ that can be obtained from Eq. (14) together with the on-mass-shell condition for the top and the $t \bar{q}$ system (here we will neglect the mass difference between the top and this system). Now, for the very classical nature of our problem, we can advocate the correspondence principle and identify $x_{\mathrm{cl}}$ with expectation value of $x(\langle x\rangle)$. Therefore, we will have

$$
\begin{aligned}
\langle x\rangle \sim x_{\mathrm{cl}} & =1-\frac{\Delta(E+p)}{E+p} \\
& =1-\frac{\Delta E}{p}=1-\frac{\beta_{1}(0) T t_{d}}{p}
\end{aligned}
$$

and this can also be written

$$
\langle x\rangle=1-\frac{T}{m_{t} \Gamma_{0}} \text {. }
$$

This is a simple and nice equation that shows in a precise way how the top fragmentation effects depend on the mass and the width of the top and on the string tension. It has also some similitude with other previous equations of the general form $\left(\langle x\rangle=1-1 \mathrm{GeV} / m_{t}\right)$ first proposed by Bjorken and later by Peterson et al. [7] Note however, than in our equation there is also an extra $m_{t}$ dependence through the width of the top $\Gamma_{0}$ and that we give a precise and meaningful expression for the dimensional constant appearing on the top mass.

\section{TOP FRAGMENTATION IN $e^{+} e^{-}, p p$, and $p \bar{p}$ REACTIONS}

In this section, we will concentrate on the production of top quarks and their related color-flux tubes in different situations of practical interest. In particular, we will consider the case of $e^{+} e^{-}, p p, p \bar{p}$ reactions because these are the ones relevant in present and future colliders. Let us start with the $e^{+} e^{-}$case. The production of the top quark in this kind of reaction is dominated by the diagram shown in Fig. 2. As the photon and the $Z$ are not colored, only a flux-tube structure is present, the antitop being the color partner of the top. Since the process is very symmetrical in this simple case, the string centerof-mass frame, the reaction center-of-mass frame, and the laboratory frame are all the same frame. In order to use Eq. (14) to compute the energy lost by the top quark we 

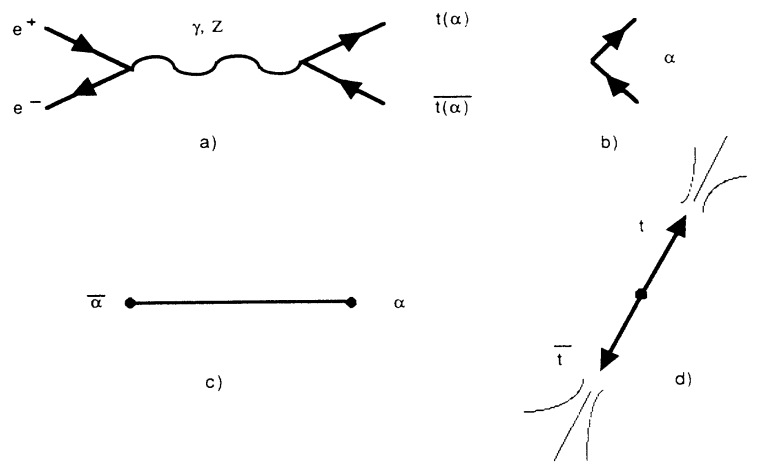

FIG. 2. (a) Lowest-order Feynman diagram of the reaction $e^{+} e^{-}$going to $t \bar{t}$. (b) The corresponding color-flux diagram. (c) Color-flux string produced. (d) Typical event signature.

have to take into account that in this equation $t_{d}$ has to be understood as the mean life of the top as measured in the string center-of-mass frame. Therefore, $t_{d}$ is given by

$$
t_{d}=\int_{0}^{t_{0}} \frac{d t^{\prime}}{\sqrt{1-\beta_{1}^{2}\left(t^{\prime}\right)}} \sim \frac{t_{0}}{\sqrt{1-\beta_{1}^{2}(0)}},
$$

where $t_{0}=1 / \Gamma_{0}$ is the mean life of the top in its proper system, $\Gamma_{0}$ being the well known decay width of the top. The last approximation applies for $t_{d} \ll p_{0} / T$, which will always be the case in this work. Therefore, our estimation of the energy lost by the top quark because of fragmentation effects when produced in $e^{+} e^{-}$reactions is

$$
\Delta E=\frac{T}{2 m_{t} \Gamma_{0}} \sqrt{s-4 m_{t}^{2}},
$$

where $\sqrt{s}$ is the energy of the reaction in the center-ofmass or laboratory frame. Of course, a similar formula applies to the energy lost by the antitop. This energy lost by the (anti)top is transferred to the hadrons produced as a result of the fragmentation process. As these hadrons will emerge roughly in the same direction as the (anti)top (the string axis), the topology of the events considered here will be the one shown in Fig. 2 .

Let us address now the more complicated case of $p p$ reactions. The relevant Feynman diagrams producing $t \bar{t}$ pairs at the partonic level are the ones of Fig. 3 (for the sake of simplicity the contribution of the sea quarks has been neglected). From the perturbative QCD point of view, all three of these channels give contribution to one unique process, i.e., the production of a $t \bar{t}$ pair with color quantum numbers $\alpha$ and $\beta$ from a pair of gluons with color-octet numbers $a$ and $b$. Therefore, the amplitude of this process will be given in an obvious notation by $M=M_{s}+M_{t}+M_{u}$.

Nevertheless, this is no longer true if one considers the color-flux tubes produced together with $t \bar{t}$ pair as an observable entity, as was done in [8]. In that case, two different final states are possible, corresponding to the two different flux-tube configurations shown in Fig. 4. In order to make contact with the ordinary perturbative computations, one can associate these two structures with the color factors $T_{\alpha \gamma}^{a} T_{\gamma \beta}^{b}$ and $T_{\alpha \gamma}^{b} T_{\gamma \beta}^{a}$ appearing in

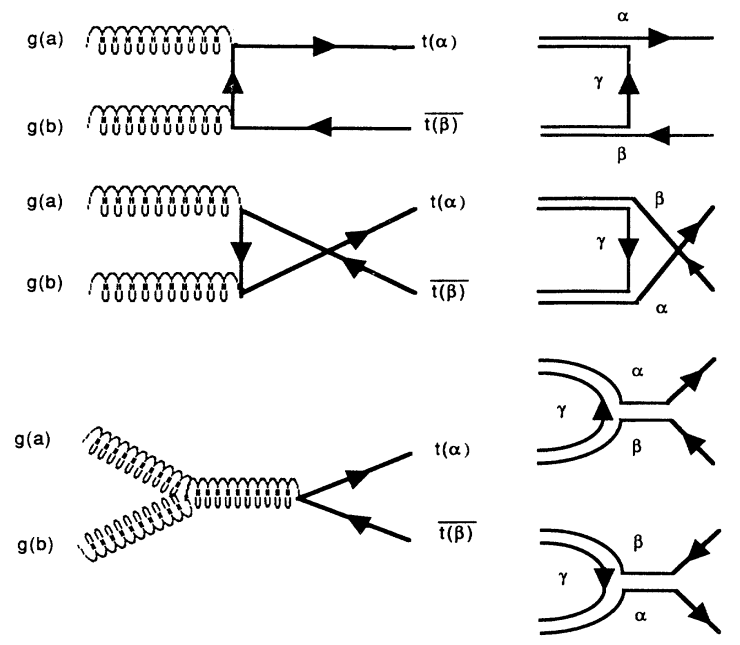

FIG. 3. Lowest-order Feynman diagrams of the process $g g \rightarrow t \bar{t}$ and the corresponding color-flux diagrams in the large- $N$ limit.

$M_{t}$ and $M_{u}$, respectively. The $s$-channel diagram $M_{s}$ is proportional to if $f_{a b c} T_{\alpha \beta}^{c}$ but because of the commutation relations of the SU $\left(N_{C}\right)$ algebra this factor can be written as $\left[T^{a}, T^{b}\right]_{\alpha \beta}=T_{\alpha \gamma}^{a} T_{\gamma \beta}^{b}-T_{\alpha \gamma}^{b} T_{\gamma \beta}^{a}$. Therefore, the amplitudes of the processes producing the structures $A$ and $B$ are

$$
M_{A}=M_{t}+M_{s}, \quad M_{B}=M_{u}-M_{s}
$$

Now it is possible to compute the corresponding cross sections for these two final states by using standard methods (nevertheless, some care is needed to properly calculate the average over gluon polarizations). The final result is

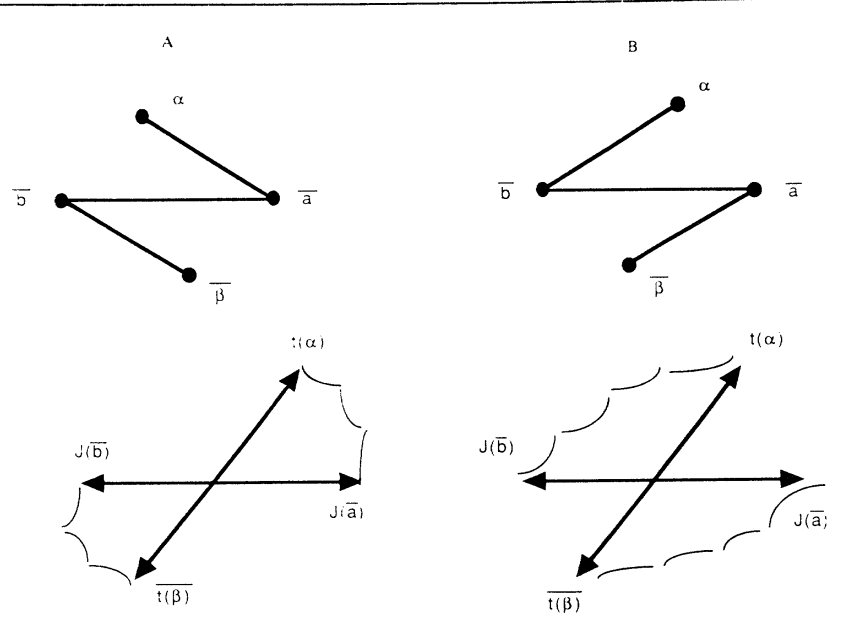

FIG. 4. Structure of the two color-flux strings that can be produced in $g g \rightarrow t \bar{t}$ reactions and their corresponding event signatures. 


$$
\begin{aligned}
\sigma_{A}=\frac{\pi \alpha_{s}^{2}}{6 \hat{s}^{2}}\left[2 \frac{\left(\hat{t}-m_{t}^{2}\right)\left(\hat{u}-m_{t}^{2}\right)}{\hat{s}^{2}}\right. \\
+\frac{\left(\hat{t}-m_{t}^{2}\right)\left(\hat{u}-m_{t}^{2}\right)-2 m_{t}^{2}\left(\hat{t}+m_{t}^{2}\right)}{\left(\hat{t}-m_{t}^{2}\right)^{2}} \\
\left.+2 \frac{m^{4}-\hat{t}(\hat{s}+\hat{t})}{\hat{s}\left(\hat{t}-m_{t}^{2}\right)}\right],
\end{aligned}
$$

$\sigma_{B}=\sigma_{A}(t \rightarrow u, u \rightarrow t)$.

Here, $\hat{s}, \hat{t}$, and $\hat{u}$ are the standard Mandelstam variables of the partonic subprocess. In fact, the sum of these two cross sections is not exactly equal to the total cross section obtained from $|\mathcal{M}|^{2}$ but the discrepancy is subleading in powers of $1 / N_{C}$. This is a well known fact which is discussed in detail in [8].

Corresponding, to the two final configurations we have two different topologies of the event. This is because, in this simple model, the hadrons appear as a result of the several breakings of the color-flux tube. In structure $A$, the produced color string goes from the top (color $\alpha$ ) to the rest of the proton after removing an $a$ gluon $\left(J_{\bar{a}}\right)$, then from this point to the rest of the other proton $\left(J_{\bar{b}}\right)$ and, finally, it arrives at the antiquark. To obtain the corresponding structure $B$ just exchange $a$ and $b$ in the above description. Therefore, in the events of class $A$, more hadron activity is expected in sectors $\left(t, J_{\bar{a}}\right)$ and $\left(J_{\bar{b}}, \bar{t}\right)$ than in sectors $\left(t, J_{\bar{b}}\right)$ and $\left(\bar{t}, J_{\bar{a}}\right)$. This situation is reversed in class $B$ events (see Fig. 4 ). The different distributions of the hadron activity in these two cases is in principle observable.

In the following, to make the problem manageable enough we will consider the color-octect indices such as $a$ or $b$ as a tensorial product of triplet-antitriplet indices. This can be understood in the framework of the large- $N_{C}$ approximation [9] which was already implicitly assumed in the derivation of Eq. (20). This approximation is equivalent to using $\mathrm{U}\left(N_{C}\right)$ instead of $\mathrm{SU}\left(N_{C}\right)$ as the color group. In Fig. 3 we display the color flow of the Feynman diagrams in this case.

With this approximation, we can consider the three pieces of the string $(\alpha \rightarrow a \rightarrow b \rightarrow \beta)$ as three colorindependent strings. Moreover, in the cases of practical interest, the energy of the rest of the protons (or antiprotons in the $p \bar{p}$ case) is much greater than the one of the top and the antitop, so we can safely assume that the velocity of the color octets does not change significantly under the effect of the central piece of the string, and remains essentially constant. We recall that in our simple model we are describing the rest of the proton after the partonic reaction as a point-particle color octet (roughly a color triplet $\otimes$ antitriplet ) of mass $m_{2} \sim 1 \mathrm{GeV}$. Therefore, we will neglect completely the effect of the central part of the string and consider only the pieces connecting the (anti)top and the corresponding rest of the proton, these two pieces being completely independent. In that case the dynamics of the strings becomes one dimensional and we can apply the results of Sec. II. Therefore, the problem is reduced to one of kinematical nature. One has to compute the energy lost by the top in the centerof-mass frame of the string connecting the top with the rest of the corresponding proton (SCM). Then a Lorentz transformation is needed to go to the center-of-mass frame of the partonic process (CM). Finally, another transformation is needed to obtain energy lost by the top in the laboratory (LAB) frame. This last transformation involves the fraction of the moment of the corresponding protons carried by the gluons, $x_{1}$ and $x_{2}$. These two parameters are random variables distributed according to the distribution functions of the gluon inside the proton $f_{g / p}(x, \hat{s})$ ( here $\sqrt{\hat{s}}$ is the energy of the partonic system). Finally, we have to take into account also that both initial-state gluons can be obtained from one proton or from the other.

Finally, we will discuss briefly the $p \bar{p}$ case. In addition to the diagrams considered in the $p p$ case, there are new contributions coming from the annihilation diagram in Fig. 5. In fact, in the case of the Tevatron, the two partonic subprocesses are roughly of the same magnitude. The diagram in Fig. 5 is the only new diagram because the contribution coming from the (anti)top inside the (proton) antiproton is neglected (we are only considering valence quarks). This diagram produces only one color structure and the related event topology is similar to the one of the structure $A$ in the $p p$ case (see Fig. 5); i.e., we expect correlations between the fragmentation of the top and the rest of the proton and between the antitop and the rest of the antiproton.

\section{NUMERICAL RESULTS}

As was mentioned in the Introduction, the aim of this paper is to give a quantitative answer to the question: are the fragmentation effects of the top quark relevant? For

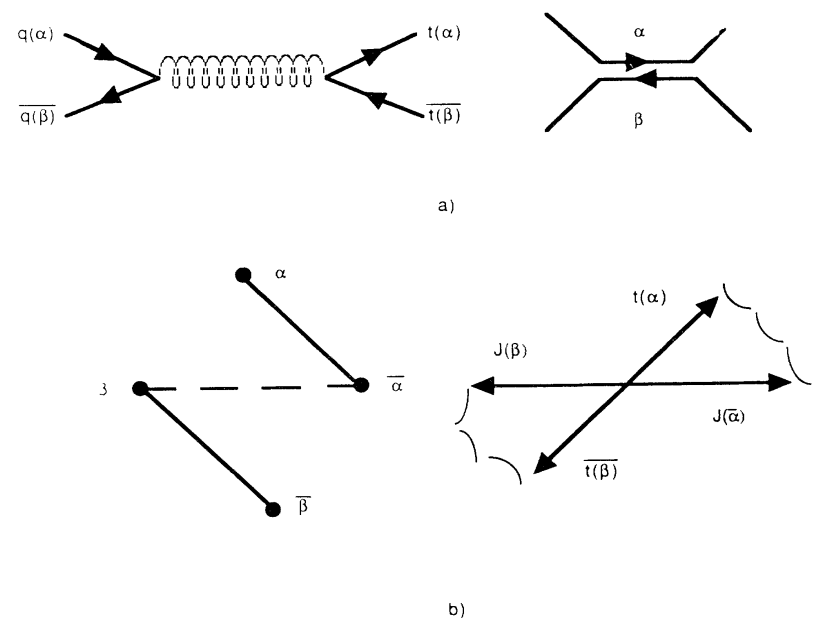

FIG. 5. (a) The dominant Feynman diagram of the annihilation $q \bar{q} \rightarrow t \bar{t}$ and its color flow lines in the large $N$ limit. (b) The produced color-flux strings and the typical signature of the event. 
this reason, in this section we compute in detail the expected energy lost by the top for this kind of effect when it is produced in different scenarios corresponding to present and next-generation colliders. In particular, we will consider the cases of a $e^{+} e^{-}$collider with center-ofmass energies of 1 and $2 \mathrm{TeV}$, two $p p$ colliders with center-of-mass energies of 16 and $40 \mathrm{TeV}$ corresponding to LHC and SSC, and finally a $p \bar{p}$ collider with center-ofmass energy $1.8 \mathrm{TeV}$ (Tevatron). For all of these cases, we compute the average lost of energy of the produced top (or antitop) in the LAB frame for different values of the top-quark mass. For the sake of completeness, we give also the average values of the rate $t_{d} / t_{h}$ for all these cases.

The way to compute these numbers has been the following. In the $e^{+} e^{-}$case, we just see the analysis of Sec. III. In the $p p$ and $p \bar{p}$ cases things are much more complicated. One of the problems is that in these cases, the energy of the parton subprocess, $\sqrt{\hat{s}}$, in the c.m. system, is not fixed but is different in each event. This is why we have to compute the average energy lost by generating a large enough number of events. Therefore we have built a Monte Carlo program to generate gluon pairs with momenta distributed according to the gluon distribution functions $f_{g / p}(x)$ of the proton, given in [10]. Then we generate the top-antitop pair with angular distributions given by Eq. (20) after making a Lorentz transformation from the LAB frame to the CM frame. Then we go to the SCM frame to compute the momentum of the top in it. The SCM frame is defined as the one in which the total momentum of the top and the related rest of the proton is zero. Only in this frame can we apply the simple Eq. (14) to compute the energy lost by the top due to fragmentation effects. Finally, note that $t_{d}$ in this equation is defined as the decay time of the top in the SCM frame, so to compute it we have to go to the rest frame of the top where the decay time is $t_{0}=1 / \Gamma_{0}$, with $\Gamma_{0}$ being

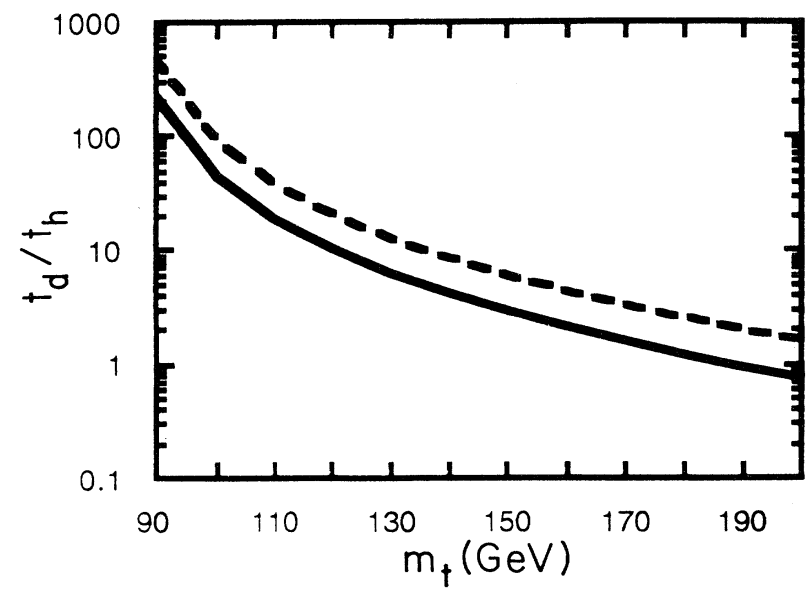

FIG. 6. The ratio $t_{d} / t_{h}$ (decay/hadronization time) vs the mass of the top in GeV in a $e^{+} e^{-}$annihilation. The solid line corresponds to a center-of-mass energy of $1 \mathrm{TeV}$ and the dashed line to $2 \mathrm{TeV}$.

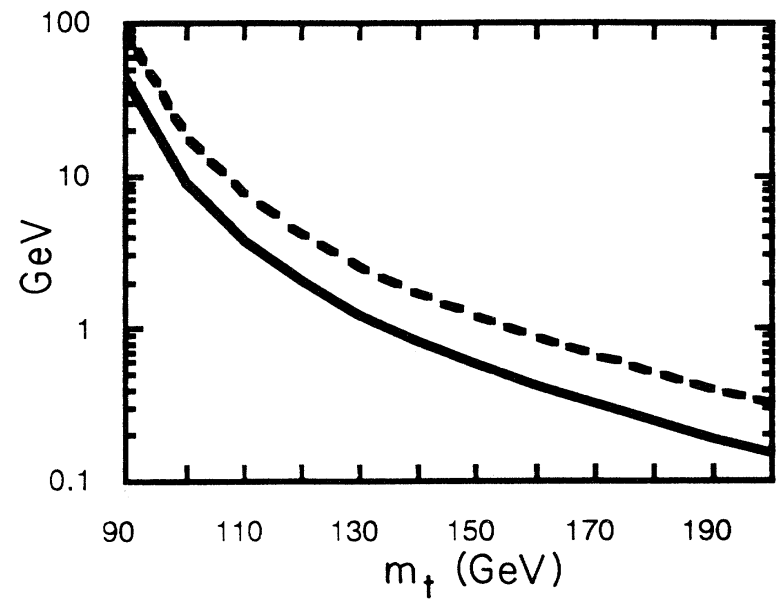

FIG. 7. Energy lost by the top quark by fragmentation effects in $\mathrm{GeV}$ vs the top mass in $\mathrm{GeV}$ in a $e^{+} e^{-}$annihilation. The solid line corresponds to a center-of-mass energy of $1 \mathrm{TeV}$ and the dashed one line to $2 \mathrm{TeV}$.

the weak decay width, and then return to the SCM frame. Therefore, the complete generation of one event requires the consideration of four different frames (LAB, CM, SCM, and the rest frame of the top).

Of course, in the $p \bar{p}$ case we have also the possibility of producing $t \bar{t}$ pairs from valence $q \bar{q}$ pairs, so we have to use also the distribution functions of the (anti)quarks inside the (anti)proton $f_{q / p}(x)=f_{\bar{q} / \bar{p}}(x)$, which also have been taken from [10].

Finally, the numerical value of $T$ has been take from Regge phenomenology. The slope of the $\rho$ Regge trajectory is given roughly by $\alpha^{\prime} \simeq 1 / 2 m_{\rho}^{2}$. On the other hand, this slope is related to the string tension through the simple relation $T=1 / 2 \pi a^{\prime}$, i.e., $T=\Lambda^{2}=m_{\rho}^{2} / \pi$, which gives a value $T \simeq(400 \mathrm{MeV})^{2}$. This number is different from

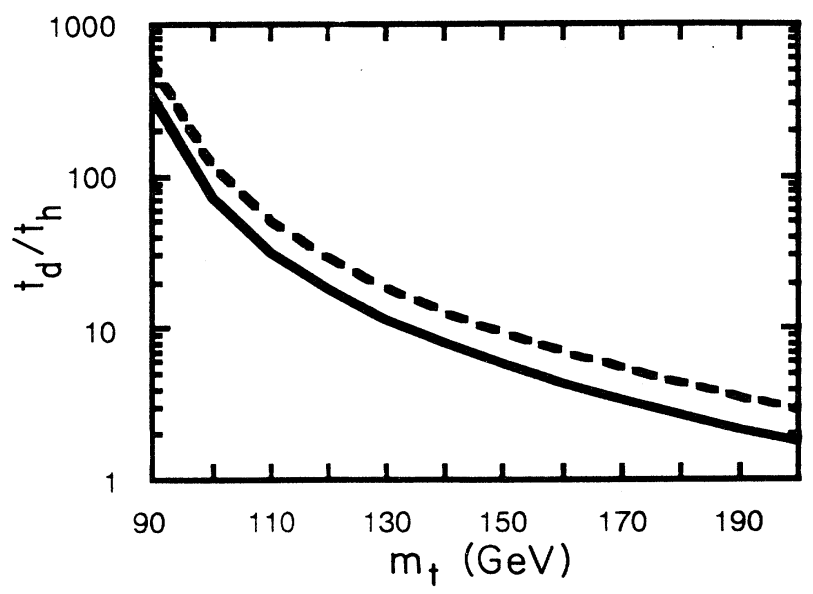

FIG. 8. Average value of the ratio $t_{d} / t_{h}$ vs the mass of the top in $\mathrm{GeV}$ in $p p$ collisions. The solid line corresponds to LHC and the dashed line to SSC. 
the one used in [3] but we consider it is more appropriate in the context of this work (see also [11]). In any case, the energy lost goes as $\Lambda^{2}$ so it is easy to trace the effect produced when a different numerical value of $\Lambda$ is advocated.

Once the energy lost by the top in the SCM frame has been computed for a single event, the program goes back to the CM and LAB frames through two more Lorentz transformations and provides the energy lost by the top quark in the LAB frame together with its energy and momentum. By averaging over a large number of events generated by our program we can compute the average or typical energy lost by the top in the LAB frame for these kinds of processes. The numerical results obtained with the program described above in different cases are shown in Figs. 6-11.
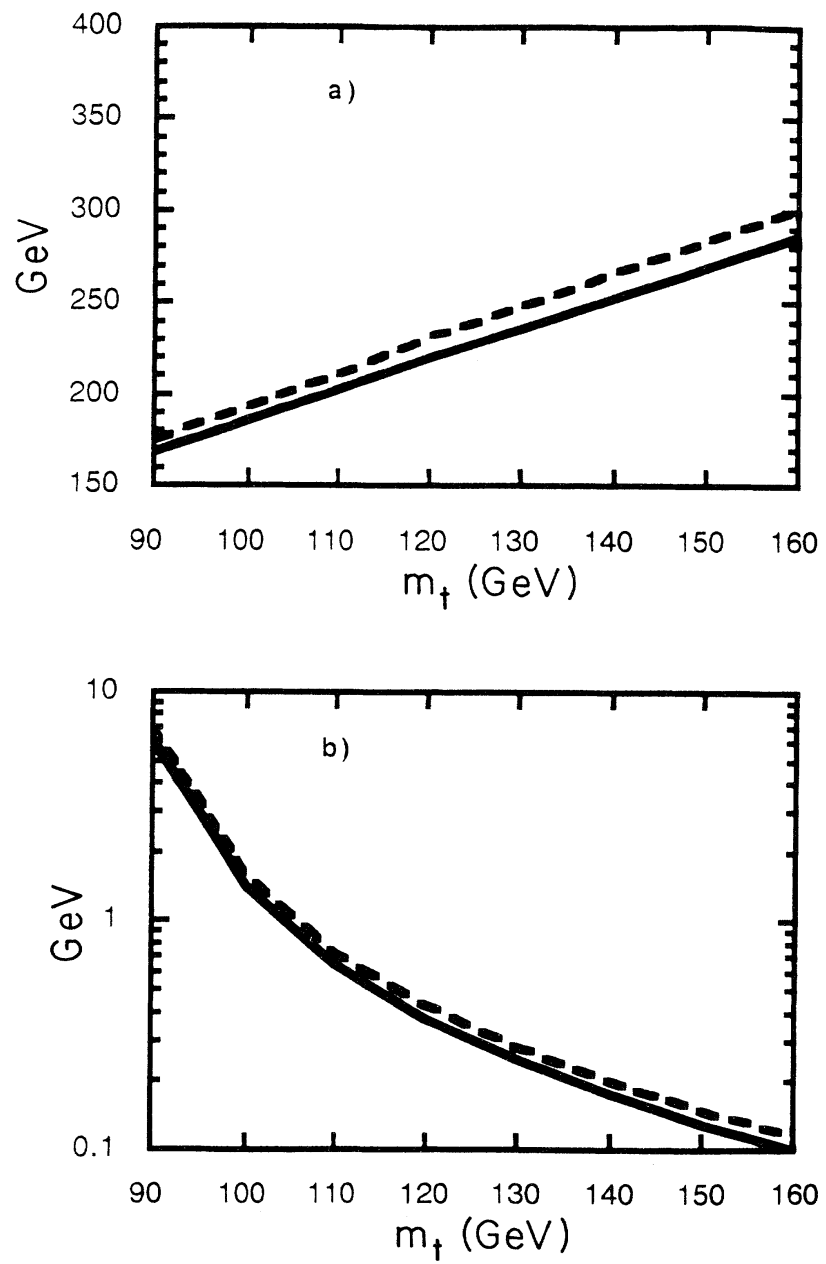

FIG. 9. (a) Mean value of the energy of the top in $\mathrm{GeV}$ as measured in the LAB frame, vs the mass of the top in $\mathrm{GeV}$ in $p p$ collisions. The solid line corresponds to LHC and the dashed line to SSC. (b) Mean value of the energy lost by the top by nonperturbative fragmentation effects in $\mathrm{GeV}$, as measured in the LAB frame, vs the mass of the top in $\mathrm{GeV}$ in $p p$ collisions. The solid line corresponds to the LHC and the dashed line to the SSC.

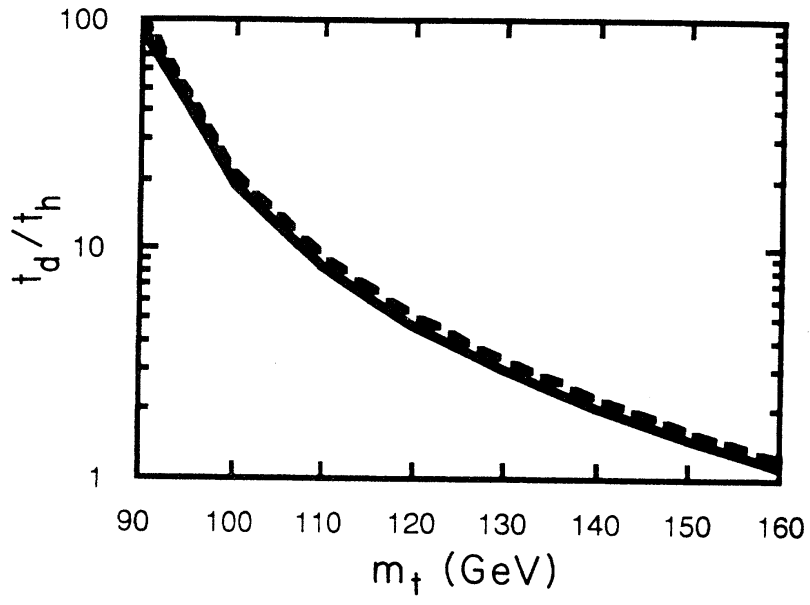

FIG. 10. Mean value of the ratio $t_{d} / t_{h}$ vs the mass of the top in $\mathrm{GeV}$ in $p \bar{p}$ annihilations. The solid line corresponds to $q \bar{q} \rightarrow t \bar{t}$ events and the dashed line to $g g \rightarrow t \bar{t}$.
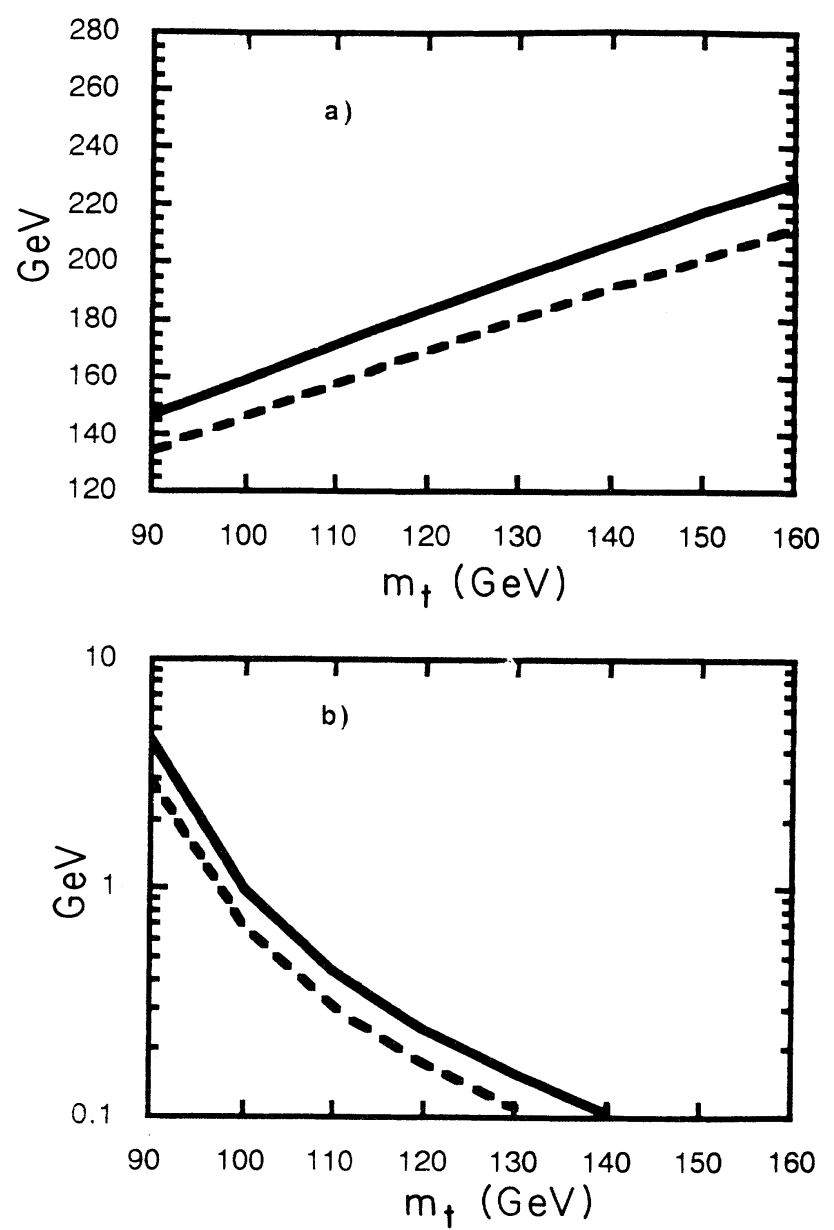

FIG. 11. Mean value of the energy of the top in GeV as measured in the LAB frame, vs the mass of the top in GeV in $p \bar{p}$ annihilations. The solid line corresponds to $q \bar{q} \rightarrow t \bar{t}$ events and the dashed line to $g g \rightarrow t \bar{t}$. (b) Mean value of the energy lost by the top by fragmentation effects in $\mathrm{GeV}$, as measured in the LAB frame, vs the top mass in $\mathrm{GeV}$, in $p \bar{p}$ annihilations. The solid line corresponds to $q \bar{q} \rightarrow t \bar{t}$ events and the dashed line to $g g \rightarrow t \bar{t}$. 


\section{DISCUSSION}

After a short inspection of these figures we realize the following simple facts. (a) As expected, the energy lost by the top quark by fragmentation effects increases with the energy of the process and decreases with the top mass. (b) In the case considered here, this energy lost is larger in the $e^{+} e^{-}$case. This probably is due to the simplicity of the kinematics and the absence of Lorentz transformations, other than the one from the top rest frame to the LAB frame. (c) The typical energy of the (anti)top produced at $p p$ colliders as measured in the LAB frame is not very different in the LHC and the SSC cases. The same happens with the energy lost by nonperturbative fragmentation effects. (d) In the Tevatron case, this energy lost is similar when the parton reaction producing the $t \bar{t}$ is $g g \rightarrow t \bar{t}$ or $q \bar{q} \rightarrow t \bar{t}$. (e) Considering that the nonperturbative fragmentation of the top is negligible for $\Delta E<1 \mathrm{GeV}$, we arrive at the conclusion that we can forget about such effects only if the mass of the top is larger than the following values: For $e^{+} e^{-}$colliders $m_{t}=130,160 \mathrm{GeV}$, for $\sqrt{s}=1$ or $2 \mathrm{TeV}$, respectively. For $p p$ colliders $m_{t}=105 \mathrm{GeV}$ for LHC and SSC and finally in the Tevatron case $m_{t}=100 \mathrm{GeV}$ for $t \bar{t}$ pairs produced through $q \bar{q}$ or $g g$ parton initial states. On the other hand, at the present value of the lower bound on the top mass $\left(m_{t} \sim 90 \mathrm{GeV}[1]\right)$, the $\Delta E$ values are 45 and 90 in the two $e^{+} e^{-}$cases considered here, $6 \mathrm{GeV}$ in the LHC and the SSC cases, and 3 or $4.5 \mathrm{GeV}$ in the Tevatron depending on the production mechanism.

Therefore, we see that fragmentation effects have some relevance if the top quark is, let us say, not heavier than $100 \mathrm{GeV}$. How could these effects appear in the detection or in the measure of the properties of the top quark? First, we note that $\Delta E$ has obviously always the same sign, i.e., the fragmentation of the top introduces a systematic (nonstatistical) error in the measure of the top mass. In particular it could modify the present and future bounds on the top mass and the confidence levels, especially if a higher bound is not set soon. The importance of this effect depends not only on the top mass but also on the precise way used to set that bound. On the other hand, it is expected in the future that the measurement of the top mass at LHC will be within an error that can be as small a $5 \mathrm{GeV}$ [12] (at the SSC we do not yet have such information). Note, however, that this is of the same order that the fragmentation effects (which are systematic) for top masses of the order of $100 \mathrm{GeV}$ or less. Therefore we think that nonperturbative fragmentation effects cannot be neglected at all in such situations.

In conclusion, there exists a window in the top-mass values in which nonperturbative top fragmentation can play a role and has to be considered seriously (more than here) if a precise measure of the top mass is required.

Note added. After this work was finished Orr was kind enough to send us a paper [13] where she extended her previous work with Rosner [3] to include the case of top produced hadronically.

\section{ACKNOWLEDGMENTS}

This work has been supported by the Ministerio de Educacion y Ciencia (Spain) (A.D.) and a CICyT project (AEN90-0034). A.D. would like to thank M. J. Herrero for bringing to his attention the interesting paper by Orr and Rosner [3] and the CERN Theory Division for its hospitality when this work was done and M.U. would like to thank P. Hernandez and J. Terron for useful discussions.
[1] CDF Collaboration, F. Abe et al., Phys. Rev. Lett. 64, 142 (1990).

[2] I. I. Y. Bigi et al., Phys. Lett. B 181, 157 (1986).

[3] L. H. Orr and J. L. Rosner, Phy. Lett. B 246, 221 (1990); 248, 474 (1990)

[4] B. Andersson, G. Gustafson, G. Ingelman, and T. Sjostrand, Phys. Rep. 97, 32 (1983).

[5] A. Chodos and C. B. Thorn, Nucl. Phys. B72, 509 (1974); W. A. Bardeen, I. Bars, A. J. Hanson, and R. D. Peccei, Phys. Rev. D 13, 2364 (1976).

[6] F. E. Low and K. Gottfried, Phys. Rev. D 17, 2487 (1978).

[7] J. D. Bjorken, Phys. Rev. D 17, 171 (1978); C. Peterson, D. Schlatter, I. Schmitt, and P. M. Zerwas, ibid. 27, 105 (1983).
[8] G. Gustafson, Z. Phys. C 15, 155 (1982); H. U. Bengstrom, Comput. Phys. Commun. 31, 323 (1984).

[9] G. 't Hooft, Nucl. Phys. B72, 641 (1974); E. Witten, ibid. B160, 57 (1979).

[10] E. Eichten, I. Hinchliffe, K. Lane, and C. Quigg, Rev. Mod. Phys. 56, 579 (1984).

[11] A. B. Migdal, Nucl. Phys. A518, 358 (1990).

[12] P. Zerwas, lecture given in 1990-1991 Academic Training Program, CERN, Geneva, Switzerland (unpublished); G. Unal and L. Fayard, Aachen, Germany, 1990, edited by G. Jarlskog and D. Rein (CERN Report No. 90-10, Geneva, Switzerland, 1990), Vol. II, p. 360.

[13] L. H. Orr, Phys. Rev. D 44, 88 (1991). 